\title{
CARACTERÍSTICAS MÉTRICAS DEL PALADAR DE NEONATOS NACIDOS A TÉRMINO Y PRE-TÉRMINO EN EL INSTITUTO NACIONAL MATERNO PERINATAL
}

\author{
Américo Munayco Magallanes ${ }^{1}$, Llisel Ocampo Escobedo², María Cortez Marino ${ }^{3}$, Maryjose Lapa Meza ${ }^{4}$
}

\begin{abstract}
RESUMEN
Objetivo: Determinar las características métricas del paladar en neonatos nacidos a término y pre-término en el Instituto Nacional Materno Perinatal (INMP). Materiales y métodos: Se realizó un estudio retrospectivo de tipo transversal en el INMP en la ciudad de Lima - Perú mediante un muestreo no probabilístico por conveniencia en 60 neonatos; divididos en dos grupos: 30 neonatos nacidos a término y 30 neonatos nacidos pre-término. El registro del paladar se hizo mediante impresiones con silicona y cubetas de acrílico individualizadas, obteniéndose modelos de estudio con yeso Ortodóncico; dichos modelos fueron llevados a Tomografía Espiral Multicorte, para realizar las mediciones longitudinales, transversales y de profundidad en vistas axiales y sagitales. Resultados: En neonatos pre-término el ancho promedio del paladar fue 26,4 $\mathrm{mm}$, mientras que en los neonatos a término fue $29,5 \mathrm{~mm}$. La profundidad promedio del paladar de los neonatos pre-término es $6,95 \mathrm{~mm}$ y en neonatos a término es $7,87 \mathrm{~mm}$. El índice palatal promedio en los neonatos pre-término es igual a 0,26 $\mathrm{mm}$ y en los neonatos a término es $0,27 \mathrm{~mm}$. El Índice Palatal no presenta diferencias significativas en neonatos a término y pre-término $(p=0,785)$. Conclusiones: La profundidad del paladar en neonatos pre-término es menor que en neonatos a término. Los neonatos a término tienen el paladar más ancho y largo que los neonatos pre-término. El índice palatal es el mismo en neonatos a término y pretérmino y, no es afectado por el género y edad gestacional.
\end{abstract}

Palabras clave: Neonato, pre-término, Índice palatal (Fuente: DeCS BIREME).

\section{METRIC CHARACTERISTICS OF THE PALATE IN TERM AND PRETERM NEWBORN IN THE NATIONAL INSTITUTE MATERNAL PERINATAL.}

\begin{abstract}
Objective: To determine the metric characteristics of the palate in neonates born at terms at the National Perinatal Maternal Institute (INMP). Materials and methods: A retrospective cross-sectional study was conducted at INMP in the city of Lima - Peru by uncier non-probabilistic for convenience in 60 neonates; divided into two groups: 30 neonates born to terms and 30 neonates born pre-terms. The palate recording was made prints with silicone and individualized acrylic buckets, obtaining study models with Orthodontic plaster; these models were taken to Espiralcorte Tomography, to perform longitudinal, transverse and depth measurements in axial and sagittal views. Results: In pre-term neonates the half width of the palate was $26.4 \mathrm{~mm}$, while in full-term neonates it was $29.5 \mathrm{~mm}$. The mean the palate of pre-term neonates is $6.95 \mathrm{~mm}$ and in term neonates is $7.87 \mathrm{~mm}$. The palatal index in pre-term neonates is equal to $0.26 \mathrm{~mm}$ and in term neonates is $0.27 \mathrm{~mm}$. The Palatal index has no significant differences in neonate terms a and pre-pre-(p-0,785). Conclusions: The basis of the palate in pre-terms neonates is lower than in full-term neonates. Term neonates have a wider and longer palate than pre-term neonates. The palatal index is the same in term and preterm neonates and is not affected by gender and gestational age.
\end{abstract}

Key words: Neonate, preterm, palatal index (Source: MeSH NLM).

\section{INTRODUCCIÓN}

A partir de los años 80 , la Odontología a nivel mundial dirige nuevamente su mirada a los niños de menor edad, principalmente a los bebés. Una muestra de ello lo constituye el programa brasilero "Un plan de atención odontológica en el primer año de vida" (1984), el cual dio paso a la creación de la "Odontología para Bebés"; sistema de atención dirigido a la promoción de la salud y prevención de la caries dental y maloclusiones; es decir, se orienta hacia la práctica de una puericultura odontológica; realizada a través de un sistema compartido entre padres y/o responsables con el odontólogo, donde se evalúan las condiciones bucales del bebé, conducta del niño, tipos de alimentación, higiene buco dental, así como el estado general del niño; para definir la situación de riesgo de caries y maloclusiones que el bebé presenta ${ }^{1}$.

Existe una clara necesidad de normas de dimensiones orales y faciales en niños dentro de este grupo de edad. Los datos de altura y peso están disponibles hasta 36 meses de edad, pero falta información sobre las

\footnotetext{
Doctor en Odontología, Especialista, Cirujano Dentista. Ex Docente FO-USMP. Docente Ordinario Facultad de Odontología-UNFV. Odontólogo Asistente del Instituto Nacional Materno Perinatal.

Mg. Salud Pública Univ. Veracruzana-México. Cirujano Dentista FO-UNFV.

3 Magister en Estomatología, Odontopediatra, Jefe Dpto. Odontoestomatología Hospital “San Bartolomé”, Docente FO-UNFV, Docente EUP-FO-UNMSM. 4 Estudiante Pregrado Facultad de Odontología-UNFV.

Citar como: Munayco A, Ocampo L, Cortez M, Lapa M. Características métricas del paladar de neonatos nacidos a término y pre-término en el Instituto Nacional Materno Perinatal. Rev Peru Investig Matern Perinat 2020; 9(3):23-7 DOI https://doi.org/10.33421/inmp.2020210
} 
dimensiones bucales ${ }^{2-4}$. La información sería valiosa para el cuidado de la salud de pacientes postraumáticos o pacientes con anomalías craneofaciales ${ }^{5,6}$. Los fabricantes también podrían beneficiarse, para el diseño de chupetes apropiados ${ }^{7}$.

La labor de identificar una de las patologías más frecuentes en la cavidad bucal como las alteraciones a nivel del paladar corresponden al médico neonatólogo y pediatra, por eso es necesario que la estomatología brinde información suficiente al pediatra y pueda diagnosticar alguna anomalía bucal e iniciar su tratamiento, con asesoría a los padres, solicitando interconsulta con el estomatólogo y brindando un tratamiento interdisciplinario ${ }^{8}$.

La región orofacial ha sido descrita como la cabina de la conciencia del término infantil ${ }^{9}$. Sin embargo; en etapas tempranas del desarrollo de la cavidad bucal los huesos blandos del paladar son maleables $y$, presiones de cualquier objeto pueden fácilmente moldear la forma del paladar ${ }^{10}$. Así, el paladar en particular puede estar sujeto a influencias tales como el modo de nacer ${ }^{11}$, posicionamiento y fuerzas gravitacionales ${ }^{12}$.

La Organización Mundial de la Salud (OMS), define parto prematuro cuando ocurre antes de las 37 semanas de gestación o cuando el peso al nacer es menor de 2500 gr. La incidencia de partos prematuros varía ampliamente entre las diferentes poblaciones $y$, es generalmente relacionado con diferencias de condiciones de vida entre países desarrollados y en desarrollo ${ }^{13}$. Al igual que otros tejidos y órganos del cuerpo, las estructuras orales y la dentición también se ven afectadas por un nacimiento prematuro.

Defectos orales tales como paladar ranurado, paladar ojival, mordida cruzada dental y, la asimetría palatina se ha reportado con frecuencias más altas en comparación con los controles a término ${ }^{14}$

El objetivo de este estudio fue determinar las características métricas del paladar en neonatos nacidos a término y los nacidos pre-término y relacionarlos según edad gestacional y género.

\section{MATERIALES Y MÉTODOS}

Este estudio es observacional, midiéndose las dimensiones del paladar tal como se registraron en los modelos de estudio, sin hacer ningún tipo de intervención; transversal, porque el registro del paladar y las dimensiones se realizaron en un solo periodo de tiempo; retrospectivo, porque se evalúa las dimensiones del paladar en recién nacidos mediante tomografía computarizada; comparativo, debido a que relaciona la información del registro del paladar según edad gestacional y género.
La muestra estuvo constituida por 60 neonatos en el Servicio de Neonatología del INMP y separados en dos grupos; grupo A: 30 neonatos nacidos a término y, grupo B: 30 neonatos nacidos pre-término.

Se consideró como criterios de inclusión a neonatos nacidos a término y pre-término en condiciones de salud estables. Se excluyeron a neonatos que presentaron desordenes neuromusculares, síndromes o malformaciones congénitas, neonatos nacidos de parto gemelar o mellizos, con dismorfismo facial, con dientes natales o neonatales. Se seleccionaron aquellos neonatos que cumplieron los criterios de inclusión y cuyos padres, luego de ser informados del procedimiento riesgos y beneficios; aceptaron y firmaron el consentimiento informado que fue presentado al comité de ética del INMP para su aprobación y ejecución. Los datos sociodemográficos y obstétricos se obtuvieron de historias clínicas. El registro del paladar se realizó en neonatos a término del servicio de Hospitalización $\mathrm{D}$ y $\mathrm{E}$ y, a los neonatos pre-término hospitalizados en los Servicio de Cuidados Intermedios Neonatales II y III que cumplieron los criterios de selección para este estudio, mediante método de observación indirecta, para el cuál se confeccionaron un stock de cubetas de acrílico para el maxilar superior de diferentes tamaños codificadas por números y debidamente esterilizadas a baja temperatura con peróxido de hidrogeno $\left(\mathrm{H}_{2} \mathrm{O}_{2}\right)$ en estado de plasma en temperatura menor de $60^{\circ} \mathrm{C}$ por un tiempo aproximado de 70 minutos. Se registró el paladar mediante impresión con silicona por condensación de alta viscosidad (Zetaplus, Putty-Zhermack ${ }^{\circledR}$ ). Utilizándose yeso Ortodóncico para los modelos de estudio y un zocalador diseñado específicamente para este estudio. Luego se ubicó los puntos de referencia mediante inspección visual, procediéndose a la corrección del paralelismo de los rebordes alveolares con respecto a los ejes $\mathrm{x}, \mathrm{y}, \mathrm{z}$ con la ayuda de un Nivel (Stanley® 8mm). Para las dimensiones del paladar se utilizaron puntos anatómicos que fueron ubicados siguiendo las referencias dadas por Procter et al. $(1998)^{15}$. Los modelos de estudios por tener estabilidad dimensional se reservaron máximo siete días para ser llevados a mediciones.

Para objeto de las mediciones, se utilizó un Tomógrafo Espiral Multicorte (TEM), marca General Electric $®$, modelo Brightspeed 16 (GE Medical Systems, Waukesha, US). Las dimensiones longitudinales y la profundidad del paladar se midieron con una herramienta del software Advantage WorksStation AW 4.3 en milímetros. El procesamiento de la información se realizó mediante un procesador Intel $\circledast$ Core (TM) i3-3110M CPU Graphics $2.40 \mathrm{GHz}$. RAM $4.00 \mathrm{~GB}$.

Sistema operativo 64 bits y, se utilizó el programa estadístico SPSS v.21. Para contrastar la hipótesis se consideró como parámetro de decisión, un margen de error del $5 \%$ con un nivel de confianza del $95 \%$. 


\section{RESULTADOS}

En la tabla 1 y Figura 1, se observa los promedios de las dimensiones longitudinales, transversales e Índice palatino en neonatos a término y neonatos pretérmino. El promedio de la profundidad, ancho y el largo del paladar en los neonatos pre-término (6.95, $26.4,12.83$ respectivamente) es estadísticamente menor que los neonatos a término $(7.86,29.52,13.65$ respectivamente) $(p<0,005)$. Sin embargo; el Índice Palatal no presenta diferencias estadísticamente significativas en neonatos a término en relación a pretérmino. $(p=0,785)$.

En la tabla 2 y figura 2, presentan los resultados del largo, ancho y profundidad del paladar y, se observa que presentan diferencias estadísticamente significativas según edad gestacional, mientras que el índice palatal no tiene diferencias significativas en cada grupo de edad gestacional.

En la tabla 3 y figura 3, se observa que el índice palatal no presenta diferencias estadísticamente significativas según el género del neonato, mientras que el largo, ancho y la profundidad promedio son diferentes significativamente entre hombres y mujeres en neonatos a término y pre término.

Tabla 1. Resultados de las características métricas del paladar en neonatos nacidos a término y neonatos nacidos pre- término.

\begin{tabular}{lccrrrrrr}
\hline & \multicolumn{3}{c}{ NEONATOS } & \multicolumn{4}{c}{ NEONATOS } \\
& PRE-TÉRMINO & \multicolumn{4}{c}{ A TÉRMINO } & \multirow{2}{*}{ * Sig. } \\
& N & X & DS & N & X & DS & \\
\hline Profundidad & 30 & 6,95 & 13,474 & 30 & 7,86 &, 880 &, 000 \\
\hline Ancho & 30 & 26,40 & 1,928 & 30 & 29,52 & 1,605 &, 000 \\
Largo & 30 & 12,83 &, 999 & 30 & 13,65 & 1,181 &, 005 \\
Índice palatal & 30 &, 2653 &, 033 & 30 &, 2677 &, 032 &, 785 \\
\hline *Prueba t & & & & & & &
\end{tabular}

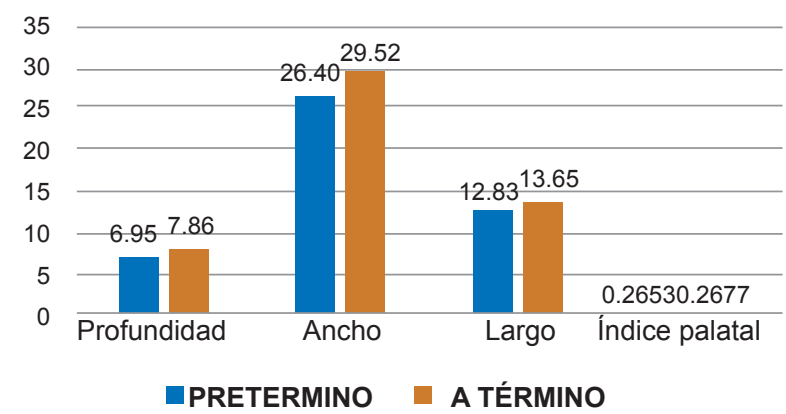

Figura 1. Características métricas del paladar en neonatos a término y pre-término.
Tabla 2. Resultados de las características métricas del paladar en neonatos según edad gestacional.

\begin{tabular}{cccccc}
\hline & $\begin{array}{c}\text { Edad } \\
\text { Gestacional }\end{array}$ & N & Promedio & DS & *sig. \\
\hline \multirow{2}{*}{ Largo } & $29-32$ & 5 & 12,94 & 1,13 & \\
& $33-36$ & 25 & 12,80 & 1,33 &, 024 \\
Ancho & $37-40$ & 30 & 13,65 & 0,68 & \\
& $29-32$ & 5 & 24,82 & 0,99 & \\
Profundidad & $33-36$ & 25 & 26,72 & 1,88 &, 000 \\
& $37-40$ & 30 & 29,5 & 0,03 & \\
& $29-32$ & 5 & 6,7 & 1,18 & \\
Índice Palatal & $33-36$ & 25 & 7,00 & 1,60 &, 001 \\
& $37-40$ & 30 & 7,86 & 0,88 & \\
& $29-32$ & 5 & 0,27 & 0,02 & \\
*kruskall wallis & $33-36$ & 25 & 0,26 & 0,03 &, 980 \\
& $37-40$ & 30 & 0,27 & 0,03 & \\
\hline
\end{tabular}

Figura 2. Características métricas del paladar en neonatos según edad gestacional.

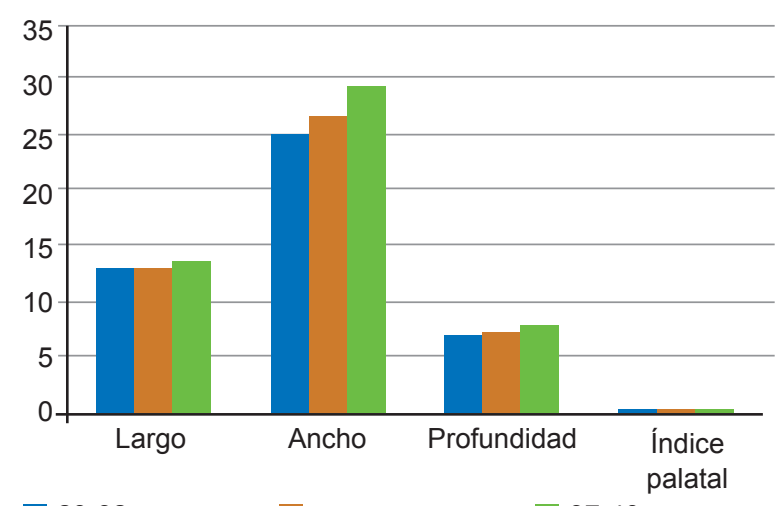

29-32 semanas

33-36 semanas

37-40 semanas

Tabla 3. Resultados de las características métricas del paladar en neonatos pre-término y a término según género.

\begin{tabular}{|c|c|c|c|c|c|}
\hline & Neonato & $\mathbf{N}$ & Promedio & DS & *Sig. \\
\hline \multirow{4}{*}{ Largo } & Pre término Masculino & 14 & 12,9 & 0,96 & \multirow{4}{*}{, 141} \\
\hline & Pre término Femenino & 16 & 12,8 & 1,06 & \\
\hline & Término Masculino & 13 & 13,3 & 1,15 & \\
\hline & Término Femenino & 17 & 13,9 & 1,16 & \\
\hline \multirow{4}{*}{ Ancho } & Pre término Masculino & 14 & 26,5 & 1,76 & \multirow{4}{*}{,011 } \\
\hline & Pre término Femenino & 16 & 26,3 & 2,11 & \\
\hline & Término Masculino & 13 & 29,6 & 1,28 & \\
\hline & Término Femenino & 17 & 29,4 & 1,84 & \\
\hline \multirow{4}{*}{$\begin{array}{l}\text { Profundi- } \\
\text { dad }\end{array}$} & Pre término Masculino & 14 & 6,7 & 0,84 & \multirow{4}{*}{,001 } \\
\hline & Pre término Femenino & 16 & 7,2 & 0,77 & \\
\hline & Término Masculino & 13 & 8,0 & 0,91 & \\
\hline & Término Femenino & 17 & 7,8 & 0,86 & \\
\hline \multirow{4}{*}{$\begin{array}{l}\text { Índice } \\
\text { Palatal }\end{array}$} & Pre término Masculino & 14 & 0,25 & 0,03 & \multirow{4}{*}{,557 } \\
\hline & Pre término Femenino & 16 & 0,27 & 0,02 & \\
\hline & Término Masculino & 13 & 0,27 & 0,03 & \\
\hline & Término Femenino & 17 & 0,27 & 0,03 & \\
\hline
\end{tabular}

* Kruskal-Wallis 


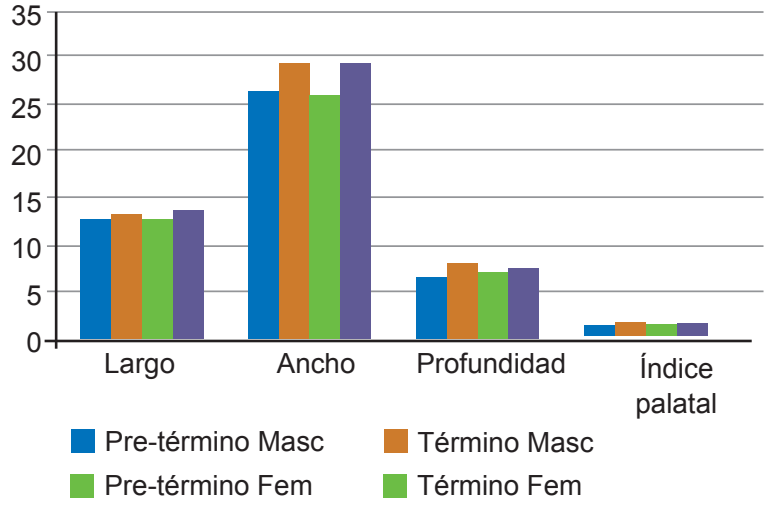

Figura 3. Características métricas del paladar en neonatos según género.

\section{DISCUSIÓN}

La investigación sobre el desarrollo del paladar se remonta a finales del siglo pasado, desafortunadamente los resultados de muchos estudios son contradictorios en varios aspectos y pueden ser difíciles de interpretar. El presente estudio realizó la descripción métrica para evaluar la configuración del paladar. Fueron tomados las dimensiones longitudinales, transversales e índice palatino y relacionadas según edad gestacional y según género.

Con respecto al largo del paladar en el presente estudio se encuentra una longitud promedio de $13.7 \mathrm{~mm}$ en los neonatos a término, y $12.8 \mathrm{~mm}$ en pretérminos, los cuales difieren con lo reportado por García et al. ${ }^{1}$ (10 mm). Así mismo Ashley- Montagu $(1934)^{16}$ reportó una longitud máxima de $25,6 \mathrm{~mm}$; esto se puede deber a que se consideraron diferentes puntos de referencia anatómicas para medir la longitud del paladar.

En cuanto al ancho del paladar, en los neonatos a término el ancho promedio es $29,5 \mathrm{~mm}$, estos resultados son muy similares a los reportados por estudios anteriores. García et al. (2011) ${ }^{1}$ encontró para neonatos nacidos a término un ancho palatino de $30 \mathrm{~mm}$. De la misma manera, Procter et al. (1998) ${ }^{15}$ reportó un ancho palatino de 28,28 $\mathrm{mm}$ en neonatos a término y Ashley-Montagu (1934) ${ }^{16}$ encontraron un ancho del paladar de $30,6 \mathrm{~mm}$.

En relación a la altura palatina, los resultados fueron heterogéneos. En neonatos pre-término el promedio de la altura palatina es $7 \mathrm{~mm}$, y en los neonatos a término es igual a 7,9 mm los cuales son muy similares a lo reportado por Ashley- Montagu (1934) ${ }^{16}$, una altura palatina de 7,6 $\mathrm{mm}$ que generalmente se encontraba sobre el centro exacto del paladar, mientras que Procter et al. (1998) ${ }^{15}$ reportó una altura palatina de 8,17 para neonatos con edad corregida de 40 semanas de gestación y García et al. (2011) ${ }^{1}$ reportó una altura palatina de $10 \mathrm{~mm}$. El paladar durante la infancia es relativamente ancho y plano ${ }^{17}$. Sin embargo; Las condiciones de cuidados neonatales que tuvieron ambos grupos durante sus primeros días de vida pueden ocasionar estas diferencias.

Con respecto al índice palatino, encontramos valores en neonatos pre-término (promedio de 0,26 ), y a término (promedio de 0,27 ), dichos resultados son relativamente similares a los presentados por Procter et al. $(1998)^{15}$ (Promedio de 0,29).

Para las características métricas del paladar según edad gestacional, el estudio reportado por Procter et al. (1998) ${ }^{15}$ en neonatos con la misma edad gestacional corregida hasta $<40$ semanas, la profundidad del paladar relativa $\mathrm{n}$ tendió a ser mayor en neonatos menos maduros, de hecho eran neonatos con mayor duración de intubación orotraqueal. Los bebés más maduros tienen los paladares más grandes, pero la gestación no tuvo ningún efecto sobre el índice palatino, es decir; la relación profundidad / anchura. Hohoff et al. (2005)3. Dichos resultados coinciden con nuestro estudio, puesto que se encontró que conforme aumenta la edad gestacional, regularmente las dimensiones del paladar también aumentan, sin embargo; dicho aumento no genera diferencias significativas en el índice palatino de neonatos pre-término y neonatos a término. Nuestros resultados también coinciden con los estudios de Esenlik et al. $(2012)^{18}$ quienes realizaron un estudio de las dimensiones del paladar en material fetal con edades entre 8- y 40 semanas de gestación y concluyeron que los índice palatal y mandibular, no cambian durante la vida prenatal.

En cuanto al desarrollo del paladar según el sexo, García et al. (2011) ${ }^{1}$ reportó que las dimensiones longitudinales y transversales del paladar tienen un promedio mayor en el género masculino, lo cual difiere con nuestro estudio, pues resultó que no existen diferencias significativas en cuanto a las dimensiones longitudinales y transversales en género masculino y femenino en neonatos nacidos a término y pre-término.

En conclusión, la profundidad del paladar en neonatos pre-término fue menor que en neonatos a término. Los neonatos a término tienen el paladar más ancho y largo que los neonatos pre-término. El índice palatal es el mismo en neonatos a término y pretérmino y, no es afectado por el género y edad gestacional.

\section{Intereses competitivos}

Los autores declaran que no tienen intereses financieros reales o potenciales competidores. Los autores no declaran intereses contradictorios que puedan influir en los resultados y conclusiones del estudio. Todos los autores tuvieron acceso a los datos y participaron en el desarrollo del estudio.

Financiamiento: Autofinanciado.

Conflicto de interés: Los autores declaran no tener algún conflicto de intereses 


\section{REFERENCIA BIBLIOGRÁFICAS}

1. García R, Bermúdez M, Rivera N, Santana $Y$, Finol A. Desarrollo del paladar en recién nacidos a término sanos y su relación con el grupo étnico. Rev De la Univer del Zulia $3^{a}$ época Ciencias Exactas, Naturales y de la Salud. 2011; 2(3):118-132.

2. Hohoff A, Rabe H, Ehmer U, Harms E. Palatal development of preterm and low birth weight infants compared to term infants - What do we know? Part 1: The palate of the term newborn. Head \& Face Medicine. 2005; 1:8. DOI: 10.1186/1746-160X1-8.

3. Hohoff, A., Rabe, H., Ehmer, U., \&Harms, E. (2005b). Palatal development of preterm and low birth weight infants compared to term infants - What do we know? Part 2: The palate of the preterm low birth weight infant. Head \& Face Medicine. 2005; 1:9. Doi: 10.1186/1746-160X-1-9.

4. Hohoff A, Rabe H, Ehmer U, Harms E. Palatal development of preterm and low birth weight infants compared to term infants - What do we know? Part 3: Discussion and Conclusion. Head \& Face Medicine. 2005; 1:10 doi: 10.1186/1746-160X$1-10$

5. Leighton BC: A preliminary study of the morphology of the upper gum pad at the age of 6 months. Swed Dent J. 1982:115-122.

6. Rubenstein L. Disscussion on: Nowak AJ, Casamassimo PS. Oral opening and other selected facial dimensions of children 6 weeks to 36 months of age. J Oral Maxillofac Surg. 1994; $52: 848$.

7. Nowak AJ, Casamassimo PS. Oral opening and other selected facial dimensions of children 6 weeks to 36 months of age. J Oral Maxillofac Surg. 1994, 52:845-847.

8. Angulo $\mathrm{M}$ y cols. El diagnóstico del pediatra ante la patología bucal benigna del recién nacido. Artículo de revisión. Acta Pediatr Mex. 2013; 34:196-204.

9. Bosma JF. Examination of the mouth and pharynx of the infant. In Proceedings: The comprehensive management of infants at risk for CNS deficits. Edited by: Heriza C. Department of Medical Allied Health Professions, University of North Carolina, Chapel Hill; 1974.
10. Palmer B: The influence of breast feeding on the development of the oral cavity. J Hum Lactat 1998, 14:93-98.

11. Pirttiniemi P, Grön M, Alvesalo L, Heikkinen T, Osborne R: Relationship of difficult forceps delivery to dental arches and occlusion. Pediatr Dent. 1994, 16:289-293.

12. Seow WK, Masel JP, Weir C, Tudehope DI: Mineral deficiency in the pathogenesis of enamel hypoplasia in prematurely born, very low birthweight children. Pediatr Dent 1989, 11:297-302.

13. Paulsson L, Bondemark L, Söderfeldt B. A systematic review of the consequences of premature birth on palatal morphology, dental occlusion, tooth crown dimensions, and tooth maturity and eruption. Angle Orthod. 2004;74(2):26979.doi:10.1043/0003-3219

14. Paulsson L, Söderfeldt B, Bondemark L. Malocclusion traits and orthodontic treatment needs in prematurely born children. Angle Orthod, 2008; 78(5):786-792. doi:10.2319/083007402.1

15. Procter M, Lether D, Oliver R, Cartlidge P. Deformation of the palate in preterm infants. Arch Dis Child Fetal Neonatal Ed. 1998; 78: F29-F32.

16. Ashley-Montagu M.The form and dimensions of the palate in the new born. Int. J. Orthodont. \& Dent for Child.1934; 20(8):810-827 New York, N.Y., USA (Abstract).

17. Bakwin \& Morris. Form and dimensions of the palate during the first year of life. Int Jr Orthodont and Oral Surg. 1936;22(10):1018-1024. New York, N. Y., USA (Summary).

18. Esenlik E, Hilal E, Şanlı G, Ali M. An Investigation Into the Morphometric Developments of the Maxillary and Mandibular Arches During the Fetal Period. The Cleft Palate-Craniofacial Journal: January 2012;49(1):60-72. doi: http://dx.doi. org/10.1597/10-238.

\section{Correspondencia:}

Américo Munayco Magallanes

Dirección: Madrid 100 Pueblo Libre

Correo: Americo_munayco@yahoo.com 